\title{
12. P.Vindob. G 30531 + 60584: Fragmente eines Philon-Codex
}

\section{(De virtutibus)*}

\author{
Tafeln XIV-XV
}

Ursula und Dieter Hagedorn (Köln)

\begin{abstract}
P.Vindob. G 30531 = P.Rainer Cent. 36, a fragment of a Greek papyrus codex published in 1983 as an unidentified Christian text, can be recognized as belonging to the treatise De virtutibus of the Jewish philosopher Philo of Alexandria. In fact, it comes from the same folio as P.Vindob. G 60584, another papyrus scrap of this treatise, which was published only a few years ago. The present article offers a new edition of the combined fragments and discusses their significance for our understanding of the medieval manuscript tradition of this specific work.
\end{abstract}

Keywords: P.Vindob. G 30531+G 60584, Philo of Alexandria De virtutibus, medieval manuscript tradition

Im Jahre 1983 veröffentlichte Kurt Treu als P.Rainer Cent. $36^{1}$ unter der Überschrift „Christlicher Ich-Bericht mit Liebesthema“ den Papyrus P.Vindob. G 30531, ein doppelseitig beschriftetes Fragment aus einem Codexblatt, welches von ihm aus paläographischen Gründen dem 4./5. Jh. n.Chr. zugewiesen wurde. Der christliche Charakter des Texts erschien ihm durch das Vorkommen einiger nomina sacra gesichert, und auf einen "Ich-Sprecher“ verwiesen mehrere Verbformen und Pronomina mit Bezug auf die 1. Person des Singulars. Eine konkretere Zuordnung war zu dieser Zeit wegen des fragmentarischen Erhaltungszustandes und zusätzlicher Textverluste auf der $\rightarrow$-Seite unmöglich.

Mit Hilfe des TLG online ist es uns jetzt gelungen, das Stück in der Schrift De virtutibus (De humanitate) Philons von Alexandria zu identifizieren, und diese Identifikation wiederum hatte die Erkenntnis zur Folge, daß P.Vindob. G 30531 aus demselben Codexblatt stammt wie P.Vindob. G 60584, der erst kürzlich von

\footnotetext{
* Wir widmen Günter Poethke diesen Aufsatz als Zeichen des Danks für eine jahrzehntelange kollegiale Zusammenarbeit und freundschaftliche Verbundenheit. Cornelia Römer, der Direktorin der Papyrussammlung der Österreichischen Nationalbibliothek, danken wir herzlich für die Bereitstellung farbiger Scans, die den Wiedergaben der Papyri auf den Tafeln XII-XIII zugrundeliegen.

${ }^{1}$ Festschrift zum 100-jährigen Bestehen der Papyrussammlung der Österreichischen Nationalbibliothek Papyrus Erzherzog Rainer, Wien, Hollinek, 1983, S. 291f. Eine Abbildung findet sich im Tafelband auf Tafel 57.
} 
Hermann Harrauer identifiziert und veröffentlicht worden ist. ${ }^{2}$ In P.Vindob. G 30531 (künftig Fr. A) ist der obere Seitenrand teilweise erhalten, P.Vindob. G 60584 (künftig Fr. B) folgt nach dem Verlust von 2 Zeilen auf der Vorderseite bzw. 3 Zeilen auf der Rückseite unterhalb davon. Wir besitzen nach der Vereinigung der Fragmente jetzt Reste der $§$ 62-66 und 67-70 der Schrift, die wir nach der Edition von Leopold Cohn zitieren. ${ }^{3}$

Was die Herkunft der Fragmente innerhalb Ägyptens anbelangt, so machte Treu zu Fr. A keinerlei Angaben, und auch der online-Katalog der Wiener Sammlung 4 bezeichnet den Abfassungsort des Stücks als unbekannt. Harrauer begründet seine Datierung von Fr. B in das 5. Jh. n.Chr., der wir uneingeschränkt zustimmen, mit der Benennung von CPR X 38 (Abb. auf Tafel 20; 420 n.Chr.) und CPR X 111 (Abb. auf Tafel 45; 418 n.Chr.) als Vergleichstexten, und da diese beide aus dem Herakleopolites stammen, erwägt er vorsichtig eine Herkunft auch unseres Philon-Papyrus von dort, fügt allerdings hinzu, daß es keine Stütze in der Fundgeschichte gebe, denn Fr. B „lag bis vor wenigen Jahren im nicht aufgearbeiteten Bestand“.

Zur Beschreibung des Erscheinungsbildes der Fragmente (Ausmaße usw.) verweisen wir auf die Ersteditionen sowie auf die Tafeln XII und XIII. Die Schrift ist eine flüssige, von der Kursiven beeinflußte Buchschrift mit zahlreichen Ligaturen zwischen den einzelnen Buchstaben. Der Schreiber verwendet nomina sacra (erhalten sind in $\downarrow$ Z. $7 \overline{\theta v}$, in Z. $17 \overline{\pi \eta \rho}$ und in $\rightarrow$ Z. $2 \bar{\theta} \bar{\omega}$ ), an Lesehilfen einen Apostroph in $\downarrow$ Z. 6 ov $\theta^{\prime}$, eine Diastole in $\downarrow$ Z. $\left.16 \varepsilon\right] \pi \alpha \gamma^{\prime} \gamma \varepsilon \lambda \lambda o \mu \varepsilon v[\omega$ und ein

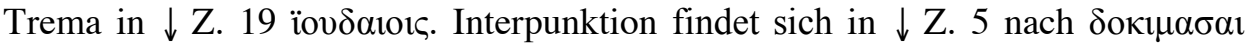
und Z. 8 nach $\theta] \varepsilon \sigma \pi[1 \zeta o v] \tau \circ \varsigma$, vielleicht auch in $\rightarrow$ Z. 16 nach $\tau[\varepsilon] \lambda \varepsilon$ เovv (vgl. den Kommentar). Eine Korrektur von vermutlich späterer Hand liegt in $\downarrow$ Z. 9 $\pi \mathrm{o \tau}] v_{1}[\mathrm{o}]^{\prime} \omega^{\prime} \mu \varepsilon v \mathrm{~s}, \mathrm{vor}$.

$\mathrm{Da}$ die $\downarrow$-Seite der $\rightarrow$-Seite vorausgeht, war schon für Harrauer offensichtlich. Beide Fragmente enthalten Teile des Außenrandes, ein Freirand ist allerdings nur auf der Rückseite vorhanden; der Umstand, daß in Fr. A auf beiden Blattseiten ein oberer Rand und damit die erste Zeile der jeweiligen Seite kenntlich ist und zur Berechnung der durchschnittlichen Breite des Schriftspiegels eine deutlich höhere Anzahl von Zeilen zur Verfügung steht, erlaubt jetzt eine genauere Rekonstruktion des ursprünglichen Seitenlayouts, als sie Harrauer möglich war, der allein auf Fr. B angewiesen war.

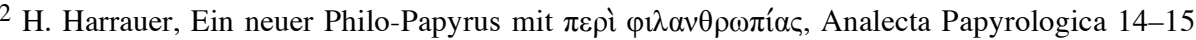
(2002-2003 [2005]) 111-115. Der Papyrus hat in der LDAB die Nummer 10370 erhalten, unter welcher künftig auch P.Vindob. G 30531 zu behandeln sein wird, der augenblichlich noch als LDAB 5851 geführt wird. Die Bedeutung des kleinen Fragments für die Philon-Überlieferung ist von James R. Royse, The Text of Philo's De virtutibus, The Studia Philonica Annual 18 (2006) 73-101, hier 96-98, gewürdigt worden.

3 Philonis Alexandrini opera quae supersunt, ed. L. Cohn, Vol. V, Berlin 1906, S. 267-335, hier 283-285.

${ }^{4}$ Augenblicklich unter http://aleph.onb.ac.at/F/?func=find-c\&ccl_term=WID\%3DRZ00002326 \&local_base=ONB08 zu finden.
} 
Auf der Vorderseite liegt die Anzahl von Buchstaben in den Zeilen, die sich einigermaßen sicher ergänzen lassen, d.h. den Zeilen 2-12 und 17-22 unserer unten abgedruckten Rekonstruktion, zwischen 46 und 55 und beträgt im Durchschnitt ca. 50,5 Buchstaben, während die Zeilen der Rückseite (Z. 1-12 und 16-22) mit einer Anzahl von 42 bis 51 und einem Durchschnitt von 46,5 Buchstaben deutlich kürzer ausfallen. Diese Differenz des Mittelwerts, die sich entsprechend auch in anderen Codices beobachten läßt, dürfte - wie man schon früher erkannt hat ${ }^{5}$ - dadurch zu erklären sein, daß der Schreiber bereits fest in das Buch eingebundene Bätter beschrieben hat; wegen des schwer zugänglichen Mittelfalzes konnte er die Breite der linken Buchseite nicht voll ausnutzen, während ihm die rechte Seite bis zum äußersten Seitenrand zur Verfügung stand. $\mathrm{Da}$ die Länge der einzelnen Zeilen jeder Seite so auffallend divergiert, liegt an der unregelmäßigen Schreibweise. Zählt man in den gut erhaltenen ersten sieben Zeilen der Vorderseite von Fr. A die Buchstabenzahl innerhalb eines ca. 6,2 cm breiten Ausschnitts, so schwankt diese schon hierbei von 19 bis 25 (durchschnittlich 22,5). Daraus läßt sich für die Vorderseite eine Breite des Schriftspiegels von nahezu $14 \mathrm{~cm}$ errechnen, für die Rückseite von ca, $12,8 \mathrm{~cm}$. Die Zeilenzahl pro Seite hat nach unserer Rekonstruktion 28 betragen. Da die Höhe der ersten 13 Zeilen der Vorderseite $8,7 \mathrm{~cm}$ beträgt, müßte der Schriftspiegel etwa $18-19 \mathrm{~cm}$ hoch gewesen sein. Zählt man einen Seitenrand von ca. $1 \mathrm{~cm}$ sowie obere und untere Ränder von je $1,5 \mathrm{~cm}$ hinzu, so ergibt sich eine Blattgröße von ca. $15 \times 21,5 \mathrm{~cm}$, was Turners „Group 7“ am nächsten kommt ${ }^{6}$. Es sei jedoch betont, daß unsere Berechnung zahlreiche Unsicherheiten birgt.

Überlegungen, welchen Umfang das vollständige Buch ehemals gehabt haben könnte, müssen mit einer Diskussion von Tintenspuren oberhalb von Z. 1 der Vorderseite beginnen, die in Treus Edition gänzlich unberücksichtigt geblieben sind; sie sind an einer Stelle erhalten, die sich einst nahe der Seitenmitte befunden haben muß, vielleicht ein wenig nach rechts versetzt. Unseres Erachtens handelt es sich dabei mit Sicherheit um Reste einer Seitenzählung; die Tatsache, daß an der entsprechenden Stelle der Rückseite keinerlei Spuren zu entdecken sind, spricht nicht gegen diese Interpretation, da auch in anderen Codices nicht selten nur die rechten Seiten mit einer Ziffer versehen wurden. ${ }^{7}$

Leider ist die Deutung der Tintenspuren nicht unproblematisch. Sie bestehen anscheinend aus drei Elementen: einer kurzen, isoliert zu sehenden Senkrechten, gefolgt dicht danach von dem Ansatz zu einer von links unten nach rechts oben verlaufenden, möglicherweise doppelt geführten Diagonalen, sowie - nach einer schmalen Lücke - dem Ende eines aus der Lücke herausragenden waagerechten Strichs. Alle Elemente zusammen können unmöglich zu nur einem einzigen Zahl-

\footnotetext{
5 Vgl. E.G. Turner, The Typology of the Early Codex, Philadelphia 1977, S. 74 mit Verweis auf T.C. Skeat.

${ }^{6}$ Vgl. Turner, Typology (wie Anm. 5), S. 19.

7 Zur Paginierung in Codices s. Turner, Typology (wie Anm. 5), S. 74-79, zur Markierung allein der rechten Buchseite s. ebenda S. 76, wo zugleich deutlich gemacht wird, daß diese Erscheinung nicht als Blattzählung anstelle von Seitenzählung mißdeutet werden darf.
} 
zeichen gehört haben, sondern es müssen drei oder zwei Ziffern gewesen sein; in letzterem Fall muß es sich bei dem Strich am Ende um eine Zahlmarkierung handeln. Wollte man mit drei Ziffern rechnen, gehörte also das erste Zeichen in die Hunderter-Reihe, dann könnte die Senkrechte am Anfang nur ein Rho gewesen sein, dessen Köpfchen oben in der Lücke verschwunden ist. Wenngleich wir diese Möglichkeit nicht gänzlich ausschließen wollen, ist sie uns doch sehr unwahrscheinlich, weil wir bei Rho einen deutlich tieferen Abstrich erwarten. Daraus folgern wir, daß das erste Zeichen der Zehner-Reihe angehört und dann ein Iota sein muß; denn kein anderes Zeichen der Zehner-Reihe wäre mit einer einfachen, isolierten Senkrechten kompatibel. Das zweite Zeichen gehört dann notwendigerweise in die Einer-Reihe; es ist am ehesten ein Alpha (erhalten ist die Spitze seines Hakens), schwerlich ein Delta oder Zeta. Wir transkribieren daher $1 \alpha^{-}$, d.h. unsere Fragmente weisen Reste der Seiten 11 und 12 des Buchs auf. $\mathrm{Zu}$ diesem Ergebnis paßt gut die Tatsache, daß die $\downarrow$-Seite der $\rightarrow$-Seite vorangeht; denn in einer Standard-Lage (4 Doppelbätter, die so geschichtet wurden, daß identische Faserverläufe aufeinander liegen; die erste und die letzte Seite sind $\rightarrow$ Seiten) ist die 11. Seite eine $\downarrow$-Seite.

Diese Schlußfolgerung ist, sofern sie denn zutrifft, von beträchtlichem Interesse für die komplizierte Überlieferung der Schrift De virtutibus und ihrer Unterteilungen. Nur eine einzige Handschrift, der Oxforder Codex $S^{8}$, über den im nächsten Absatz mehr zu berichten sein wird, tradiert nämlich die Schrift in derselben Abfolge, wie wir sie heute in der Edition von Cohn lesen, d.h. mit den Teilen a. §§ 1-50 De fortitudine, b. §§ 51-174 De humanitate, c. §§ 175-186 De paenitentia und d. §§ 187-227 De nobilitate. Andere Handschriften haben eine divergierende Abfolge, enthalten vielfach nicht alle Teile und verbinden diese gelegentlich mit anderen philonischen Schriften. ${ }^{9}$ Berechnet man nun, wie viel Platz alles, was in Cohns Fassung der Schrift De virtutibus dem Einsatz unseres Papyrus vorausgeht, in diesem eingenommen haben müßte, so kommt man zu dem Ergebnis, daß mehr als 12 Seiten zu erwarten wären. ${ }^{10}$ Der Papyrus kann also, wenn die erste der uns vorliegenden Seiten wirklich die 11. Buchseite ist, nicht dieselbe Abfolge wie S gehabt haben, d.h. nacheinander die Teile a. und $b$. von De virtutibus. Der Anfang von De humanitate allein hätte andererseits viel zu wenig Platz eingenommen, nämlich nur etwa 2 1/3 Seiten. ${ }^{11}$ Uns erscheint daher unausweichlich, daß im Papyruscodex die Abhandlung De humanitate auf eine andere Schrift gefolgt ist als De fortitudine - vorausgesetzt, die Grundlage unserer Berechnung ist solide und der Papyrustext wies keine anderen Irregularitäten auf wie etwa größere Auslassungen.

\footnotetext{
${ }^{8}$ Oxoniensis Seldenianus 12 aus dem X./XI. Jh.

${ }^{9} \mathrm{Zu}$ Einzelheiten verweisen wir wieder auf den Aufsatz von J.R. Royse (wie Anm. 2), bes. S. $77-81$.

${ }^{10}$ Die beiden Seiten des Papyrus nach unserer Rekonstruktion nehmen in Cohns Ausgabe rund 2850 Schriftzeichen (ohne Satzzeichen usw.) in Anspruch, eine Seite also rund 1425 Zeichen; alles, was bei Cohn vorausgeht, beläuft sich auf ungefähr 17670 Zeichen. $17670: 1425=12,4$.

${ }^{11}$ Es handelt sich um ca. 3300 Zeichen.
} 
Aber auch unabhängig von dieser speziellen Frage hat der Papyrus unter überlieferungsgeschichtlichem Aspekt beträchtlichen Wert, bestätigt er doch an mehreren Stellen Lesarten der bereits erwähnten Handschrift S; diese nimmt in $D e$ virtutibus insofern eine Sonderstellung ein, als sie bei sehr zahlreichen Gelegenheiten allein gegenüber der restlichen handschriftlichen Tradition das Richtige bewahrt hat. ${ }^{12}$ Der spektakulärste Fall ist zweifellos $\rightarrow$ Z. 5 ov̉ -8 ö $\psi \varepsilon \sigma \iota v$, wo der Papyrus mit $\mathrm{S}$ einen längeren Passus bezeugt, der ansonsten überall fehlt. Ein Zusammengehen des Papyrus mit S findet sich ferner an folgenden Stellen (mit Fragezeichen kennzeichnen wir die Fälle, in denen wir die Lesung des Papyrus



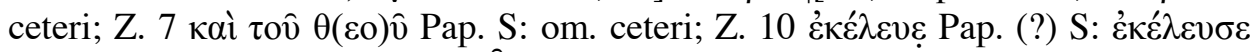

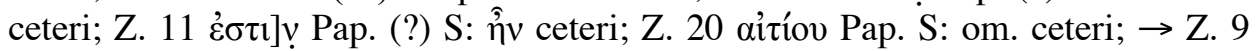

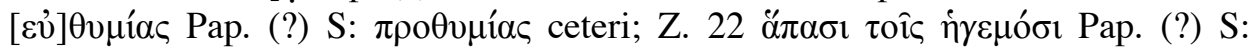

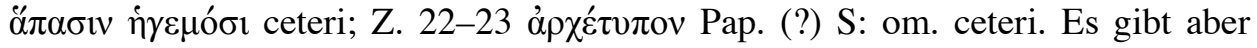
auch Belege dafür, daß umgekehrt der Papyrus gegen S mit den ceteri geht: $\downarrow$ Z. 1

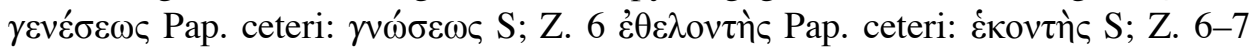

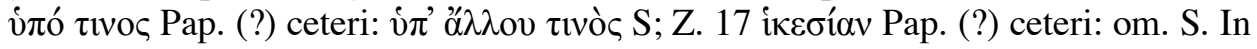
$\rightarrow$ Z. 16 findet sich eine bemerkenswerte Übereinstimmung des Papyrus mit dem Zeugen $\mathrm{G}^{1}$. Eine von der handschriftlichen Überlieferung abweichende Sonderles-

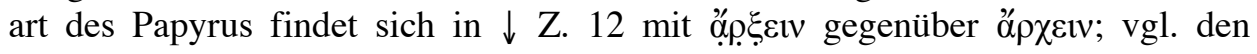
Zeilenkommentar.

Wir drucken im folgenden eine hypothetische Rekonstruktion beider Papyrusseiten. Es versteht sich von selbst, daß überall da, wo die Fragmente keinen Seitenrand bewahren, und zumal in den vollständig ergänzten Zeilen die exakte Stelle der Zeilenwechsel nicht zu bestimmen ist.

\footnotetext{
12 Zur komplizierten Überlieferung der Schrift De virtutibus und ihrer Teile und zur speziellen Bedeutung von S vgl. L. Cohn in den Prolegomena zu Vol. V seiner Edition (zu S besonders S. VIII-X); dens., Neue Beiträge zur Textgeschichte und Kritik der Philonischen Schriften, Hermes 43 (1908) 177-219 (zu De virtutibus und der Rolle von S bes. S. 210-215); D.T. Runia, Underneath Cohn and Colson: The Text of Philo's De virtutibus, in: ders., Philo and the Church Fathers. A Collection of Papers (Suppl. to Vigiliae Christianae 32), Leiden u.a. 1995, S. 75-101 (zu S bes. S. 93-95); J.R. Royse (wie Anm. 2), zu S bes. S. 94-96. Zu den verwendeten Handschriftensiglen vergleiche man Cohns Prolegomena, S. 266, und Runia, S. 83.
} 
$\downarrow$-Seite

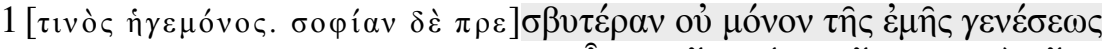

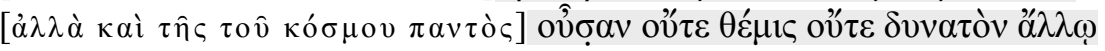

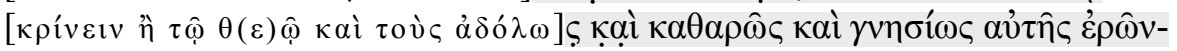

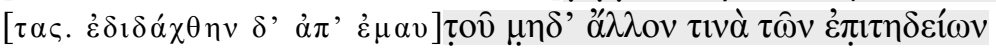

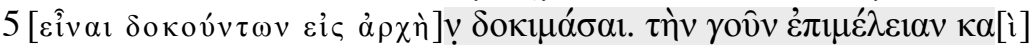

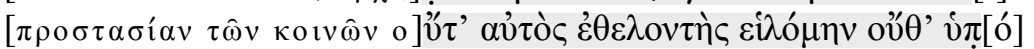

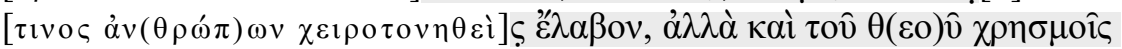
$\hat{\varepsilon}[v \alpha \rho-]$

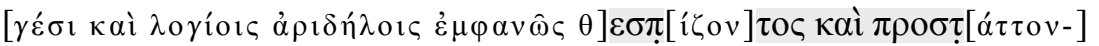

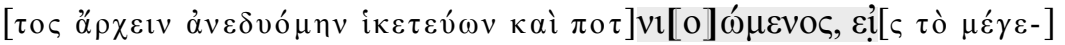

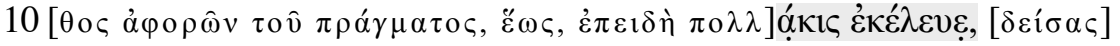



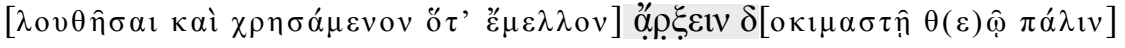

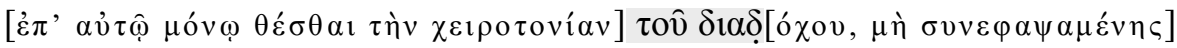

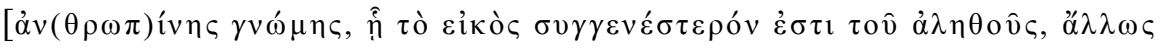
$\tau \varepsilon \kappa \alpha \grave{\imath} \tau \hat{\eta} \varsigma]$

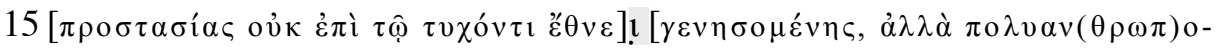
$\tau \dot{\alpha} \tau \omega \mu \dot{\varepsilon} \nu]$

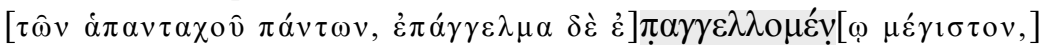

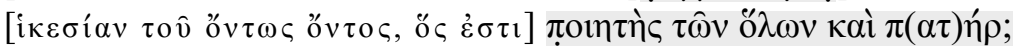

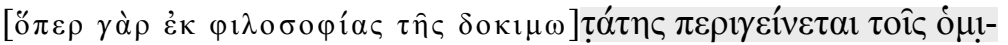

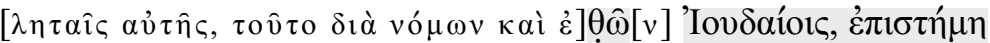

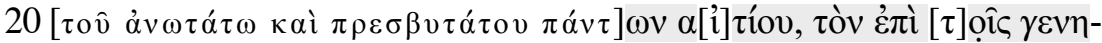

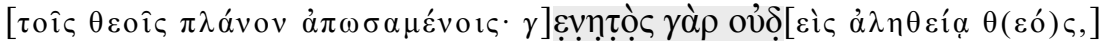

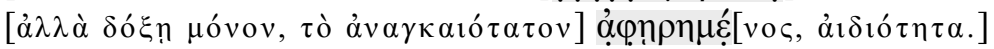

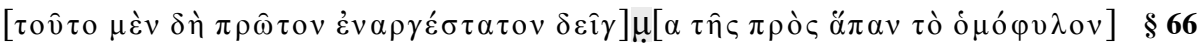

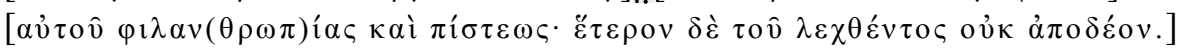

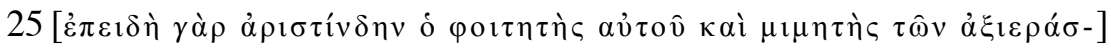

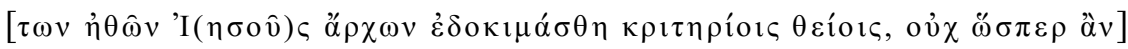

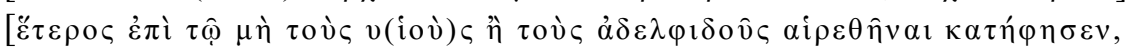
$\dot{\alpha} \lambda \lambda \dot{\alpha}]$

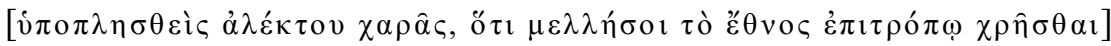

$\rightarrow$-Seite

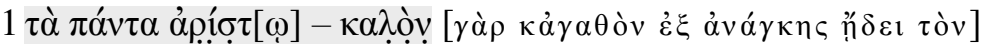

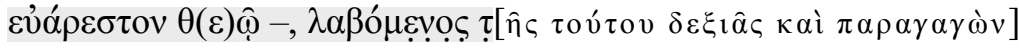

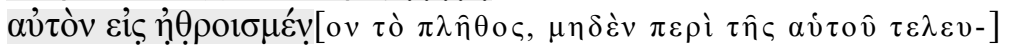

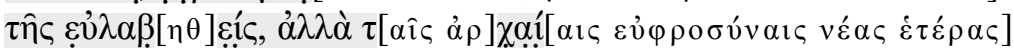

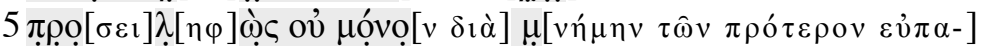

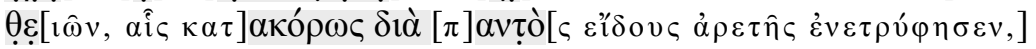

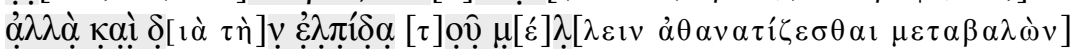




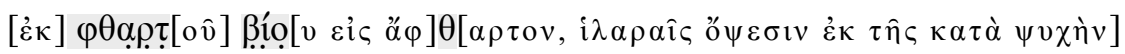

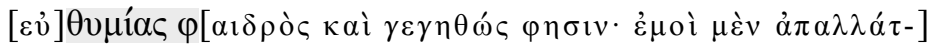

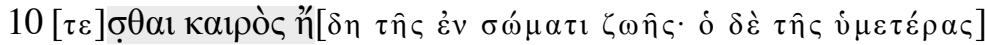

$[\dot{\varepsilon}] \pi ı$ I

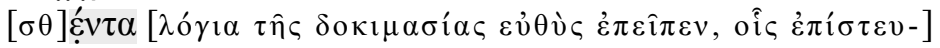

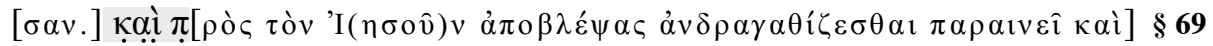

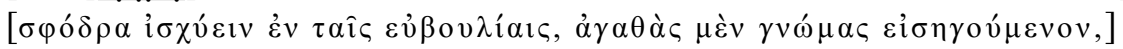

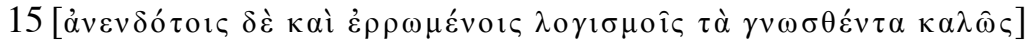

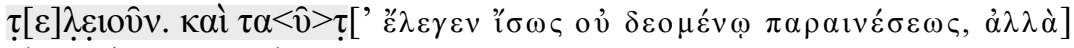

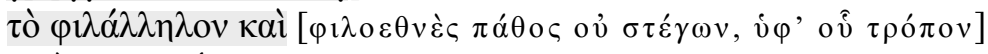

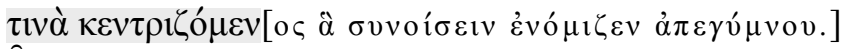



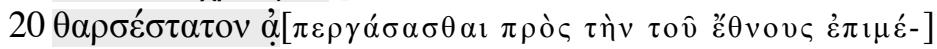

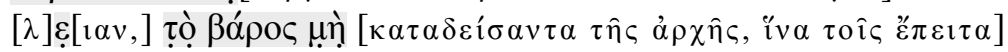

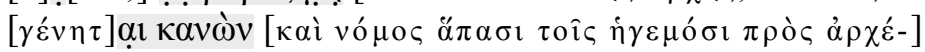

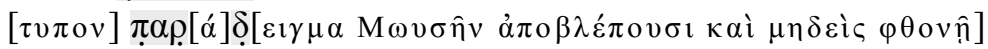

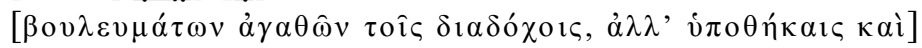

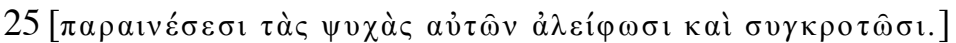

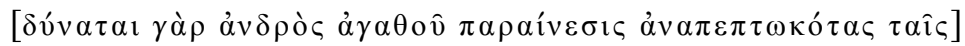

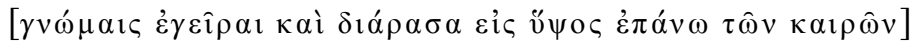

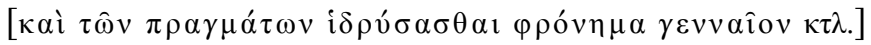

\section{$\downarrow$-Seite}

o Zur Lesung der Seitenzahl s. die Einleitung.

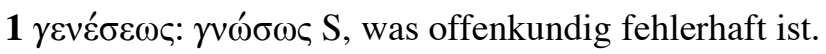

2 ov̂oav: Man meint, an der Stelle des $\sigma$ eine tief nach unten reichende Haste


oủpavôิ $\tau \varepsilon$ ?

oひ้ $\varepsilon$ : Das Tau steht in Korrektur. Der Schreiber hat vielleicht einen Ansatz zu

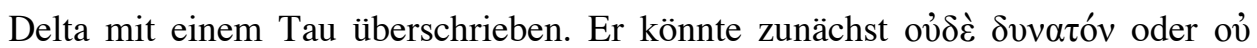

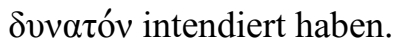

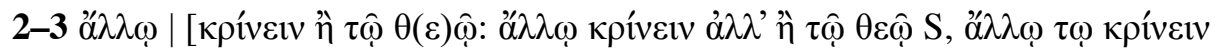
ì $\tau \hat{\varphi} \theta \varepsilon \hat{\varphi}$ ceteri. Aus Platzgründen haben wir eine die Überlieferungen von $S$ und der ceteri kombinierende Rekonstruktion gewählt, die den kürzest möglichen Text ergibt.

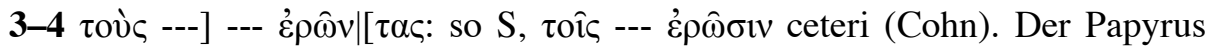
kann offenkundig nicht den Text der ceteri gehabt haben; wir folgern daher, daß er die Lesart von $\mathrm{S}$ vertreten hat. Der Akkusativ ist als Ergänzung zu dem Infinitiv крíveıv durchaus akzeptabel.

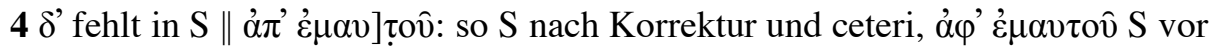

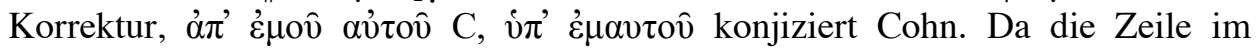


Papyrus ohnehin recht kurz ausfällt und aus stilistischen Gründen eine Partikel zu erwarten ist, wird man auf $\delta$ nicht verzichten. Man könnte sogar darüber spekulieren, ob $\delta \varepsilon ́$ im Papyrus in scriptio plena geschrieben war und der Papyrus möglicherweise auch die Lesart von $\mathrm{C}$ hatte.

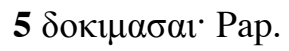

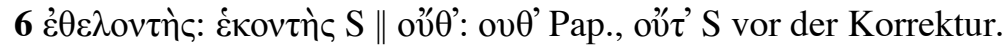

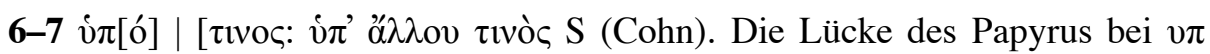
beginnt im Buchstaben Pi, so daß ein eventueller Apostroph nicht sichtbar würde. Hätte der Papyrus die Lesart von S gehabt, müßte Z. 6 nach $v \pi-$ wegen der Trennungsregeln - mindestens noch $\alpha \lambda$ enthalten haben, wodurch diese Zeile weiter nach rechts reichte als die vorangegangenen. Außerdem müßten auch in dem verlorenen Teil von Z. 7 mehr Buchstaben Platz gefunden haben als in dem entsprechenden Raum der vorhergehenden Zeile. Wir haben es daher vorgezogen, den Text der Vulgatüberlieferung zu übernehmen.

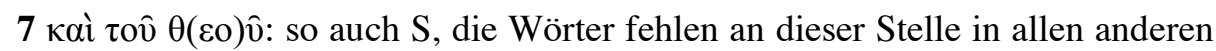

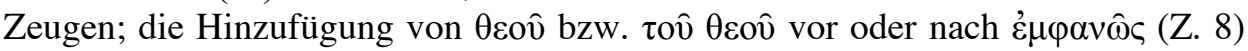
in einzelnen Handschriften beruht offensichtlich auf Konjektur.

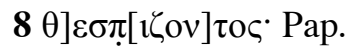

9 In der Lücke am Zeilenfang sind weniger Buchstaben ergänzt, als nach dem verfügbaren Platz zu erwarten wären. Der Papyrus könnte also eine Textabweichung gehabt haben.

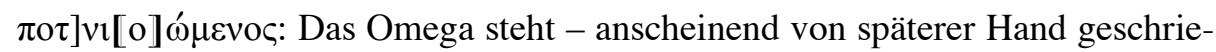
ben - oberhalb des durch einen Querstrich getilgten Omikron.

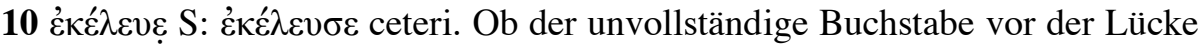
im Papyrus ein Epsilon oder ein Sigma ist, läßt sich nicht mit Sicherheit entscheiden, doch spricht unseres Erachtens mehr für ersteres.


Platzgründe.

12 ộ $\xi_{\varepsilon} \varepsilon v$ : Dies ist - jedenfalls nach Cohns kritischem Apparat zu urteilen eine singuläre Lesart des Papyrus gegenüber ő $\rho \chi \varepsilon ı v$ in der gesamten mittelalterlichen Tradition. Die ersten drei Buchstaben sind zwar unvollständig erhalten, aber doch zuversichtlich gelesen; für $\xi$ besitzen wir im Papyrus kein weiteres Beispiel, doch passen die Reste vorzüglich, während $\chi$ uns ausgeschlossen erscheint.

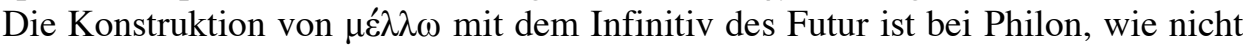

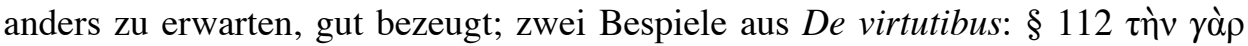

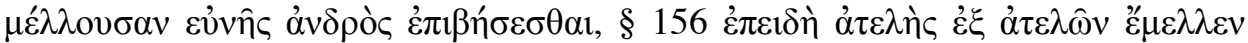

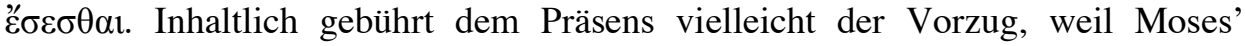
Führerschaft zu diesem Zeitpunkt unmittelbar bevorstand.

14-15 Die beiden Zeilen sind in unserer Rekonstruktion mit 60 und 59 Buchstaben deutlich länger als die durchschnittlich ca. 51,5 Zeichen enthaltenden vorangehenden Zeilen. Dieselbe Textmenge statt auf zwei Zeilen auf drei verteilt 
ergäbe jedoch um so viel zu kurze Zeilen, daß diese Lösung erst recht nicht in Frage kommt. Wenngleich der Papyrustext sehr unregelmäßig geschrieben ist und die Dichte der Buchstaben generell schwankt, liegt doch die Vermutung nahe, daß hier mit einer Auslassung zu rechnen ist. In der mittelalterlichen Tradition findet sich dafür allerdings kein klarer Anhaltspunkt; die uneinheitliche Überlieferung des Passus $\grave{\eta}$--- $\dot{\alpha} \lambda \eta \theta$ ov̂ in Z. 14, wo wir Cohns Fassung (nach S) folgen, bietet kaum Varianten in der Textlänge, abgesehen von der vollständigen Auslassung in $\mathrm{G}^{1}$.

15 c $\theta v \varepsilon]$ !: Die Unterlänge des Iota, welches Harrauer nicht transkribiert hat, ist

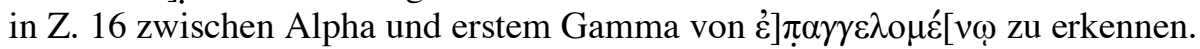

$16 \varepsilon] \pi \alpha \gamma^{\prime} \gamma \varepsilon \lambda \lambda \circ \mu \varepsilon \varepsilon$ [ $\omega$ Pap. laut Harrauer; auf der Abbildung ist die Diastole nicht (mehr?) erkennbar. Zur Verwendung der Diastole zwischen Doppelkonsonanz vgl. z.B. E. G. Turner, Greek Manuscripts of the Ancient World, London ${ }^{2}$ 1987, S. 11 mit Anm. 50, und F. Th. Gignac, A Grammar of the Greek Papyri of the Roman and Byzantine Periods I, Milano 1975, S. 162-165. Am Ende der Zeile 16 ist noch reichlich Platz für $\mu \varepsilon ́ \gamma ı \sigma \tau o v$, was Konsequenzen für die folgende Zeile hat.

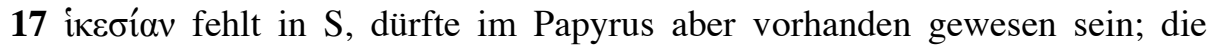
Lücke zu Beginn der Zeile würde, da unseres Erachtens $\mu$ ć $\gamma$ ббov noch in Z. 16 Platz gefunden hat, sonst nicht hinreichend gefüllt.

18-20 Im Gegensatz zu Harrauer sind wir der Meinung, daß in dieser Partie die Zeilenenden des Papyrus erhalten sind; in Z. 19 und 20 ist unseres Erachtens deutlich ein Freiraum erkennbar. Hier sollten also keine Buchstaben mehr ergänzt werden.

18 Lies $\pi \varepsilon \rho \gamma^{\prime} v \varepsilon \tau \alpha$.

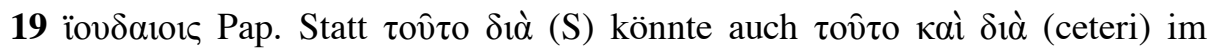
Papyrus gestanden haben.

$20 \alpha[i] \tau$ íov: so auch $\mathrm{S}(\mathrm{Cohn})$, die übrigen Hss. lassen das Wort aus.

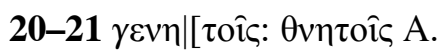

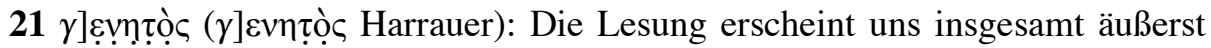
problematisch. Von Epsilon, Eta und Tau sind nur minimale Spuren erkennbar. Vor dem sicheren Schluß-Sigma sehen wir eher ọ̣.

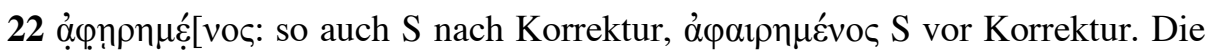
Endung des Worts schwankt in anderen Handschriften.

$23 \delta \varepsilon i \gamma] \mu[\alpha$ : Das unseres Erachtens hinreichend deutliche My ist bei Harrauer unberücksichtigt geblieben.

$\rightarrow$-Seite

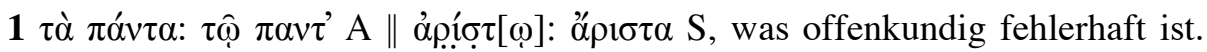
Die Lesung des Papyrus bleibt unsicher; falls nach dem Tau noch geringe Tinten- 
spuren vorhanden sein sollten, was sich an der Abbildung nicht klar entscheiden läßt, wären sie mit Alpha besser kompatibel als mit Omega.

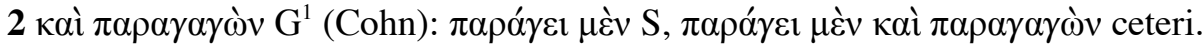
Die Langfassung der Vulgata ist für den Papyrus aus Platzgründen auszuschliessen, während die Lesung von $S$ auch hier vorgelegen haben könnte.

5 ov - 8 ó $\psi \varepsilon \sigma ı v:$ Dieser Passus fehlt in der Vulgatüberlieferung, ist also ausschließlich in S erhalten.



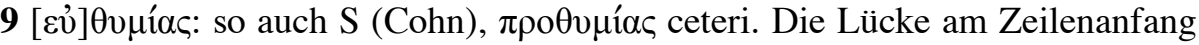
enthält kaum Platz für drei Buchstaben.

11 Oberhalb des ersten Pi von $\dot{\varepsilon}] \pi ! \imath \uparrow \rho \pi[\hat{\eta} \varsigma$ meinen wir Tintenspuren zu sehen, die wir nicht erklären können.

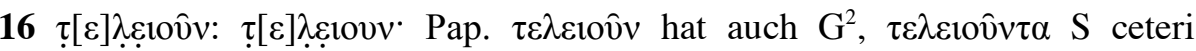
(Cohn). In der editio princeps von P.Vindob. G 60584 hat H. Harrauer den schwach erkennbaren Punkt nach dem Ny als Abkürzungszeichen oder eventuell nur dessen Rest interpretiert und entsprechend $[\tau \varepsilon] \mid \lambda \varepsilon เ o v v(\tau \alpha)$ hergestellt; die mit dieser Annahme verbundenen Schwierigkeiten hat er ausführlich dargelegt. Es dürfte sich jedoch an dieser Stelle, wo ein Satz endet, eher um eine Interpunktion

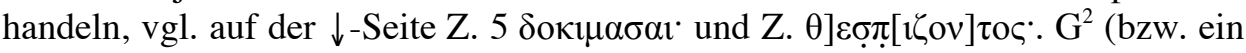
Vorfahr) hat $\tau \varepsilon \lambda \varepsilon 10 \hat{v} v$ offenbar in einer Reihe mit den Infinitiven $\alpha \nu \delta \rho \alpha \gamma \alpha \theta i \zeta \varepsilon \sigma \theta \alpha$ i

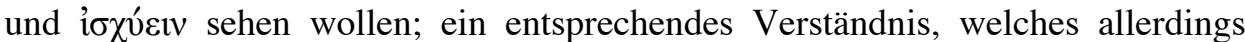
durch die Korrelation von $\mu \varepsilon \dot{v} v$ und $\delta \varepsilon ́$ in Z. 14/15 als falsch erwiesen wird, könnte auch schon früher einmal - d.h. in der Tradition, die der Papyrus repräsentiert aufgetaucht sein.

$\tau \alpha<\hat{v}>\tau[$ [: Ein Ypsilon nach $\tau \alpha$ erscheint uns ausgeschlossen. Am ehesten sieht man ein leicht hochgestelltes Tau. Der Schreiber hat das Ypsilon also anscheinend ausgelassen; denn eine andere Ergänzung der Stelle bietet sich nicht an.

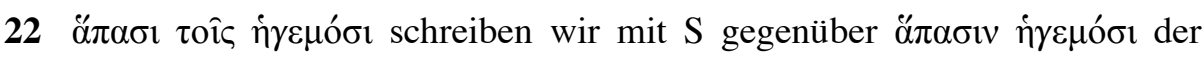
ceteri (Cohn), weil dadurch die Buchstabenzahl der Zeile besser dem Durchschnitt angenähert wird. Das Argument ist aber keineswegs zwingend.

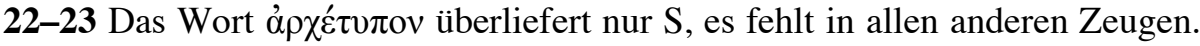

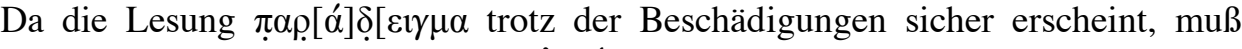
auch der Papyrus aus Platzgründen ả $\rho \chi \varepsilon ́ t v \pi o v$ enthalten haben. 